\title{
Cervical Venous Vascular Anatomy for Ventriculoatrial Shunt Applications: Anatomical Study and Surgical Approach Recommendation
}

\author{
Baris OZONER ${ }^{1,2}$, Ebubekir AKPINAR ${ }^{1,3}$, Abuzer GUNGOR ${ }^{1,4}$, Akin AKAKIN ${ }^{1,5}$, Turker KILIC ${ }^{1,5}$ \\ ${ }^{1}$ University of Bahcesehir, School of Medicine, Prof. Rhoton Anatomy and Surgery Training Center, Istanbul, Turkey \\ ${ }^{2}$ University of Health Sciences, Kartal Dr. Lutfi Kirdar Research and Education Hospital, Department of Neurosurgery, Istanbul, Turkey \\ ${ }^{3}$ University of Health Sciences, Basaksehir Cam and Sakura City Hospital, Department of Neurosurgery, Istanbul, Turkey \\ ${ }^{4}$ University of Health Sciences, Bakirkoy Research and Training Hospital for Neurology, Psychiatry, and Neurosurgery, Department of \\ Neurosurgery, Istanbul, Turkey \\ ${ }^{5}$ University of Bahcesehir, School of Medicine, Department of Neurosurgery, Istanbul, Turkey
}

Corresponding author: Baris OZONER drbarisozoner@gmail.com

\section{ABSTRACT}

AIM: To develop an approach for atrial application of ventriculoatrial (VA) shunts after revealing the venous anatomy with facial and cervical anatomical dissections.

MATERIAL and METHODS: Five cephalic cadavers were used in the study. Facial and cervical regions of the cephalic cadavers were examined with layer by layer anatomical dissection. Venous angiography and ultrasonography were performed to obtain additional data on the cervical venous vascular anatomy. Subsequently, we developed an approach for atrial catheter applications.

RESULTS: No anatomical variations were detected in the dissections. The common facial vein, which was formed by the facial vein and retromandibular vein, was observed to drain into the internal jugular vein. As a result of dissections and examinations, an incision approximately $2 \mathrm{~cm}$ below the mandible, extending from the projection of the submandibular notch to the trace of the angulus mandible, was considered adequate to expose the common facial vein for atrial catheter insertion.

CONCLUSION: The approach described in our study is appropriate for the application of an atrial catheter for VA shunts. Revealing the venous anatomy with examinations contributes to the success of the operation.

KEYWORDS: Hydrocephalus, Common facial vein, Ventriculoatrial shunt, Operative technique

ABBREVIATIONS: CSF: Cerebrospinal fluid, VP: Ventriculoperitoneal, VA: Ventriculoatrial, MR: Magnetic resonance

\section{INTRODUCTION}

$\mathrm{V}$ Tentricular dilatation, a result of impaired balance in the formation and absorption of cerebrospinal fluid (CSF), is defined as hydrocephalus (16). Various surgical techniques have been defined to reroute excess CSF in the ventricular system to other cavities that have absorption capacities, such as the pleura (6), peritoneum (25), and right atrium (19). Of these, ventriculoperitoneal (VP) shunt interventions are the most preferred methods $(4,19)$. However, VP shunt applications are not suitable for cases such as peritonitis, intraabdominal malignancy, peritoneal absorption failure and previous multiple VP shunt malfunctions $(18,20)$.

Ventriculoatrial (VA) shunt systems, which ensure CSF drains from the ventricular system to the right atrium, are an eligible
Baris OZONER (1D): 0000-0003-0534-2766

Ebubekir AKPINAR (1) : 0000-0002-4069-0021

Abuzer GUNGOR (D) : 0000-0002-2792-7610
Akin AKAKIN (1) : 0000-0003-2093-4073

Turker KILIC (1) : 0000-0001-5982-9700 
option when VP shunting is inapplicable $(18,20)$. Although they are barely regarded as a first-line option in hydrocephalus, VA shunt applications continue in daily practice (18). However, the application of the atrial catheter of the VA shunt comprises some difficulties. The familiarity with the anatomy of this region has decreased among neurosurgeons due to decreased application numbers in the last decades. Also, this procedure involves complication risks such as malposition, obstruction, shunt infection, endocarditis, heart failure, intra-atrial thrombus, and pulmonary hypertension $(3,10,13)$. The literature on standardized surgical approach recommendations to minimize the complication risks of the procedure is scarce.

Venous vascular anatomy can be revealed with radiological examination methods. One of the non-invasive radiological examination methods is cervical venous angiography, which can be performed with magnetic resonance (MR) imaging without contrast administration (8). Also, ultrasonography is reported to be beneficial for obtaining information about the venous vascular structure of the neck (24).

In light of this information, a standardized approach consolidated with preoperative radiological examinations could decrease complications. Therefore, in this study, we aimed to define a reliable surgical approach for VA shunt atrial catheter application using cadaveric dissection for venous vascular anatomy and non-invasive examinations, such as venous angiography and ultrasound.

\section{MATERIAL and METHODS}

This study was evaluated and approved by the Clinical Research Ethics Committee of Bahcesehir University (date: 02/09/2020, number: 2020-11/09). Cervical and facial dissections were carried out on formaldehyde-preserved human cadavers. The dissection was performed using basic microsurgical instruments. Five cephalic specimens were examined in the study. All cadavers were of Caucasian ethnicity. None of the cadavers studied had previous surgery or disease that affected the integrity of the cervicofacial anatomy.
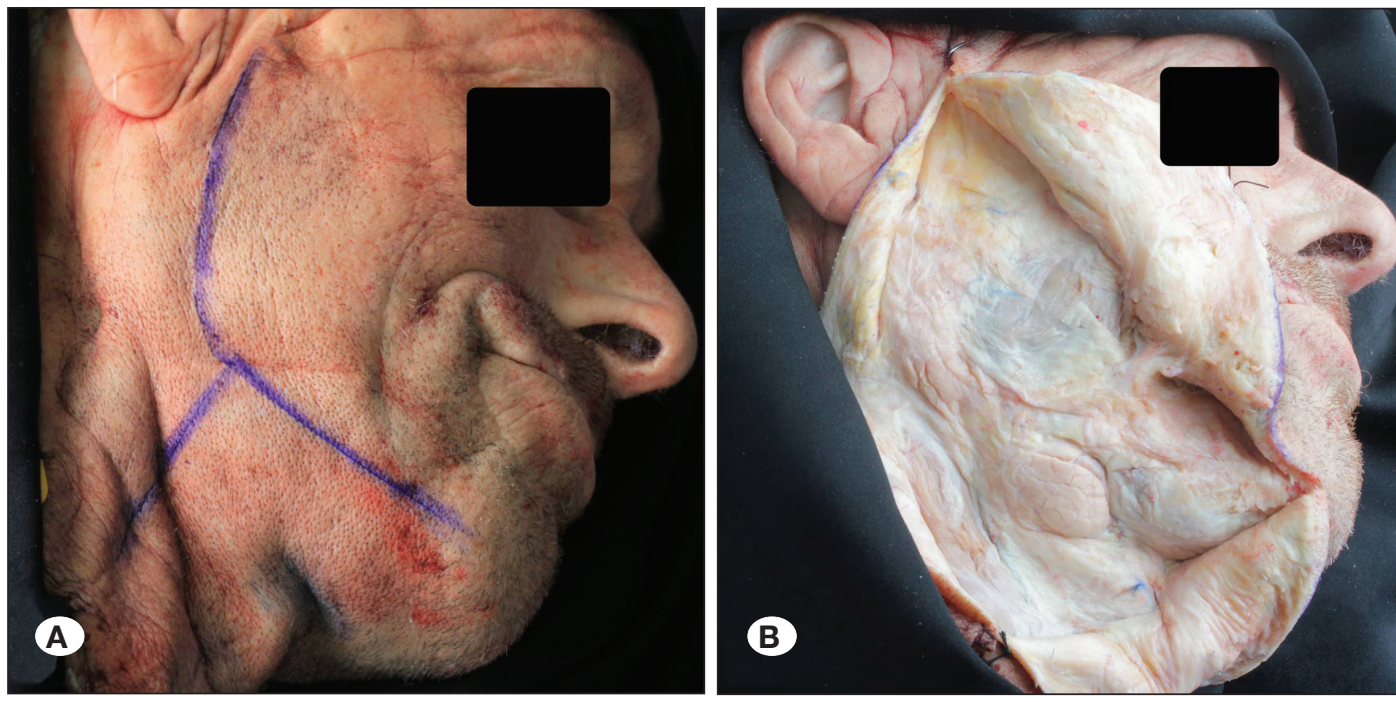

Figure 1: Facial and cervical anatomical dissection to observe the venous vasculature. A) A skin incision was used that allows cervical and facial anatomical dissection. B) After skin and subcutaneous tissue dissection, before the platysma muscle dissection, the submandibular gland and parotid gland were observed. 

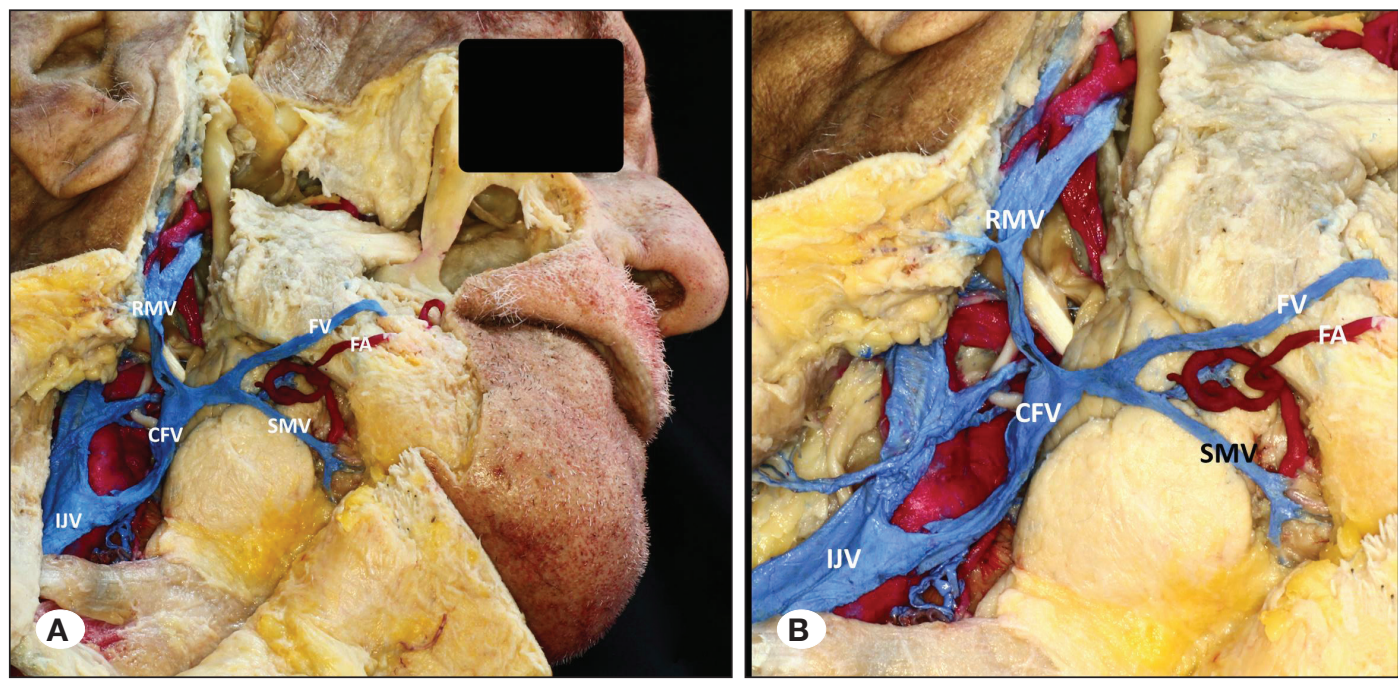

Figure 2: Facial and cervical anatomical dissection for the venous vasculature.

A) Venous vasculature after the platysma muscle and deep dissections; the parotid gland was removed and the sternocleidomastoid muscle was suspended. B) Magnified view of the venous vasculature. CFV: common fascial vein; FA: fascial artery; FV: fascial vein; IJV: interanl jugular vein; RMV: retromandibular vein; SMV: submental vein.

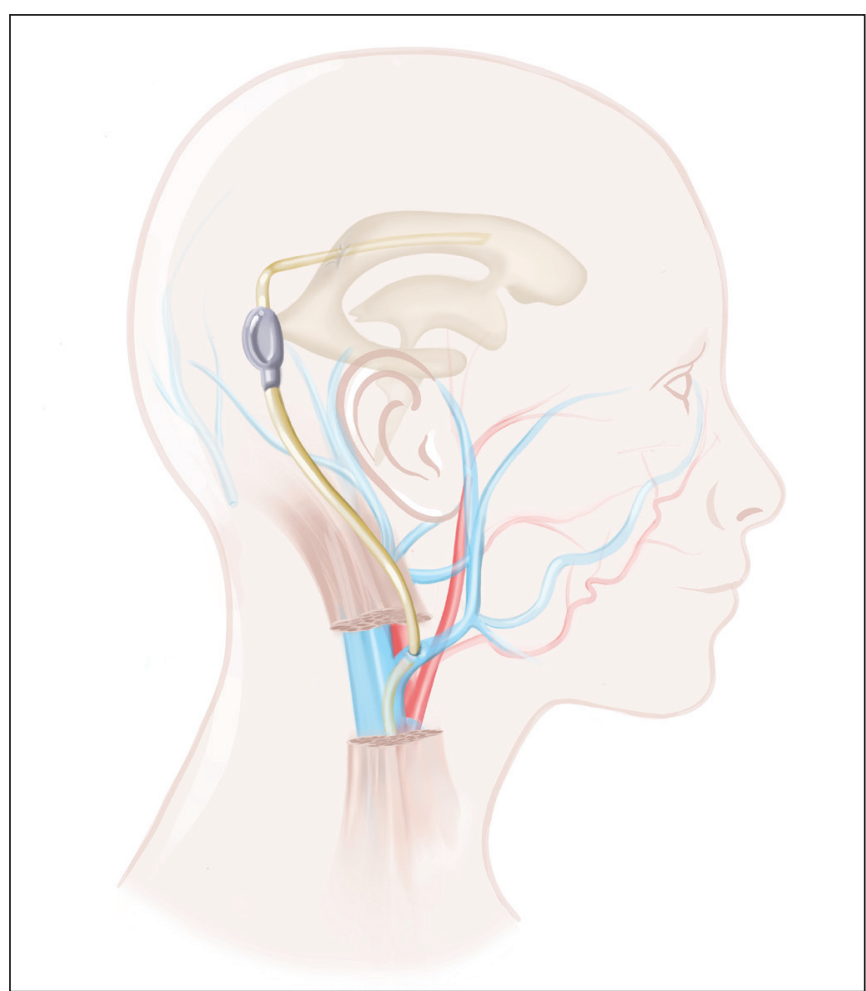

Figure 3: Illustration of the recommended surgical approach.

was observed behind the groove for the facial artery in all cadaveric specimens while passing over the lower border of the mandible. In all specimens, the facial artery descended anterior to the facial vein. After that, the facial vein passed beneath the platysma and entered the submandibular triangle in the anterior cervical region. In this region, the facial vein received the submental vein and passed over the lateral or medial surface of the superficial part of the submandibular gland. Then, the facial vein descended, pierced the deep fascia, and received the retromandibular vein to form the common facial vein.
The submental vein originated from the submental triangle in the anterior cervical region. Then the submental vein crossed laterally to enter the submandibular triangle. Consequently, the submental vein passed over the superficial part of the submandibular gland and drained into the facial vein.

In the parotideomasseteric region, the retromandibular vein was formed in the posterior of the parotid gland, descended, and then divided into two branches (anterior and posterior) at the inferior end of the parotid gland. The posterior branch of the retromandibular vein crossed to the sternocleidomastoid region and formed the external jugular vein over the superficial surface of the sternocleidomastoid muscle.

In the carotid triangle, the facial vein and anterior branch of the retromandibular vein formed the common facial vein. The common facial vein descended posteriorally to the submandibular gland, crossing the carotid arteries and draining into the internal jugular vein. With this course, the common facial vein received the superior thyroid veins.

\section{Surgical Approach Proposal for Atrial Catheter Applications}

Using the information obtained from venous vascular anatomical dissections, a recommended surgical approach was developed. An illustration of the recommended surgical approach is shown in Figure 3. Anatomical dissections made in this approach are presented in Figure 4A, B. While performing the approach on cadaveric specimens, two reference points on the mandibular bone were considered: the groove for the facial artery and the angulus mandibula. Both anatomical markers were easily palpated. The planned surgical incision was parallel to the mandibular corpus and approximately $2 \mathrm{~cm}$ below the mandibular corpus. The incision was approximately $3.5 \mathrm{~cm}$ in length and extended from the projection of the groove for the facial artery to the projection of the angulus mandible. Venous vascular structures were encountered in the submandibular and carotid triangle after the skin incision and subcutaneous and platysma dissections. The facial vein and submandibular vein that formed the common facial vein were identified. The common facial vein was considered to be the 

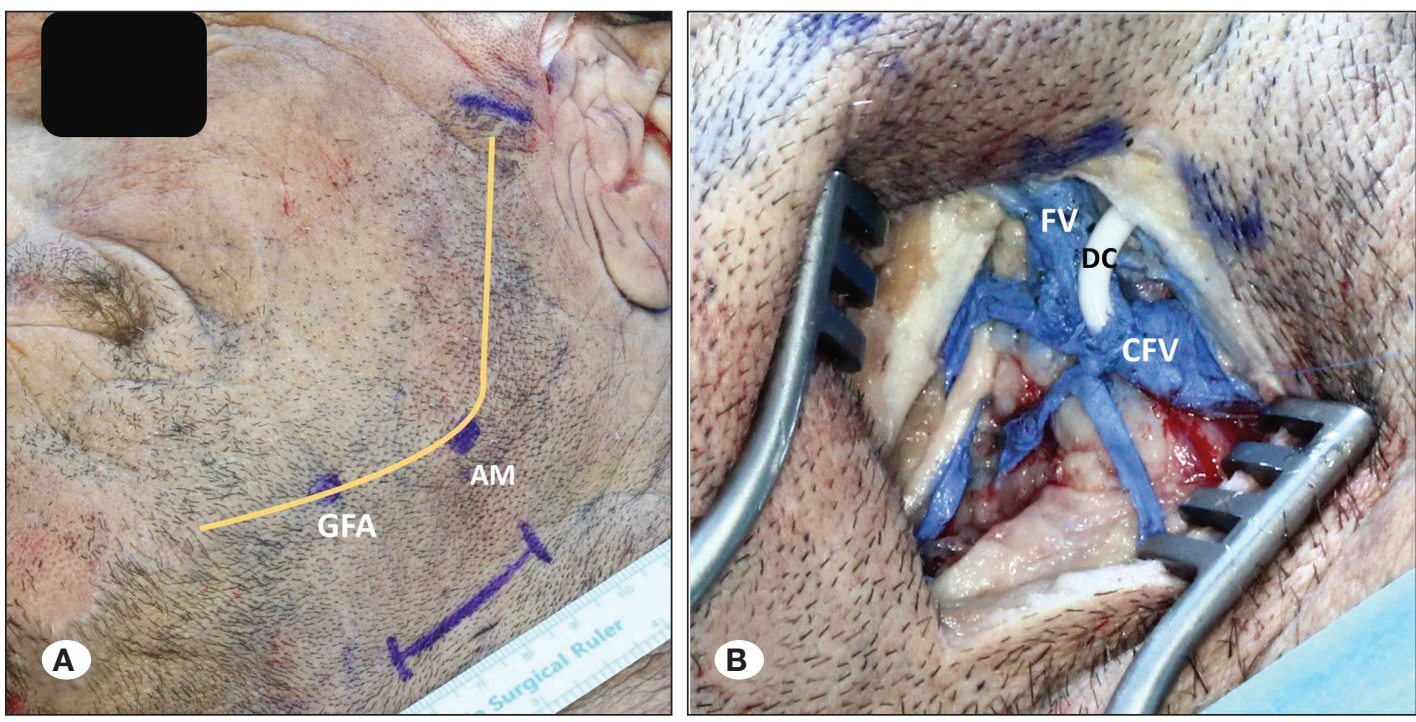

Figure 4: Recommended surgical approach

A) Skin incision is shown for insertion of the distal atrial catheter of the ventriculoatrial shunt. B) Appearance after the skin and subcutaneous and platysma muscles are dissected and the distal atrial catheter is inserted through the common facial vein. AM: angulus mandibula; CFV: common facial vein; DC: distal catheter; FV: facial vein; GFA: groove for facial artery

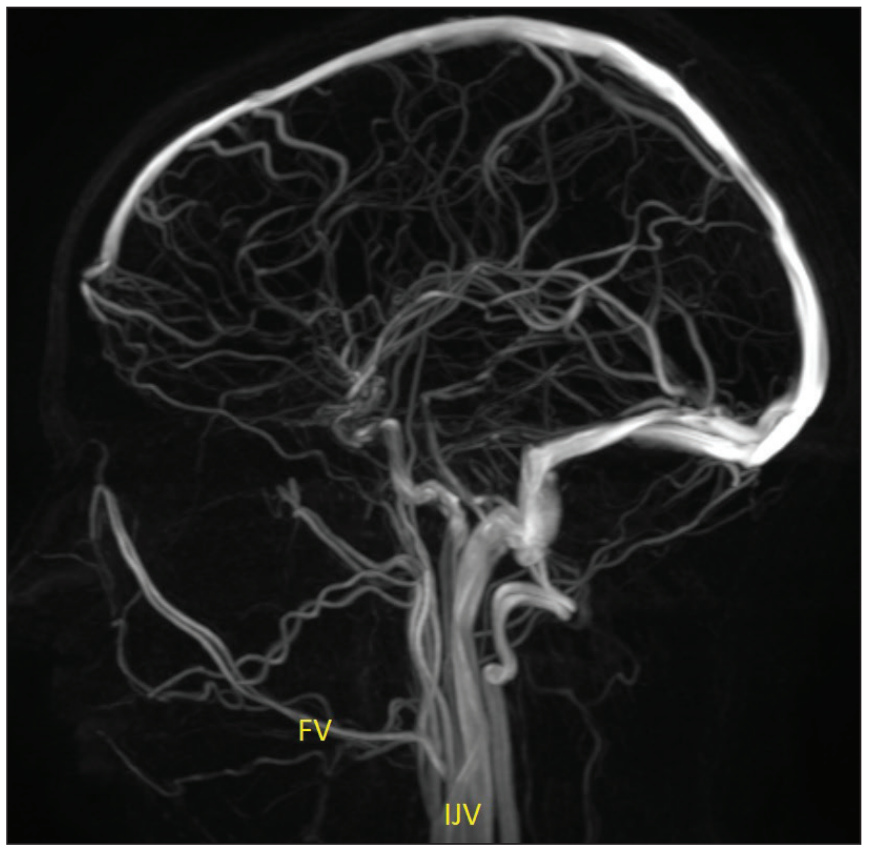

Figure 5: Venous magnetic resonance angiography showing the cranial and cervical venous vasculature; the facial vein (FV) joins with the retromandibular vein to form the common facial vein that drains into the internal jugular vein (IJV).

most appropriate venous structure for application of the atrial catheter.

In ventricular catheter placement via the posterior approach using Frazier's point, the trocar that was sent subcutaneously through the posterior incision reached the submandibular incision effortlessly. The trocar was removed following the atrial catheter passing through the trocar. Then, the atrial catheter was inserted using an incision on the common facial vein.

\section{Magnetic Resonance Venography}

A sample for MR venography is presented in Figure 5. The relationship between the facial vein, submandibular vein, and common facial vein with the jawline could be identified in MR venography of individuals with normal venous vasculature. According to the results of venographies, there was no need for modification of the incision in the recommended approach for atrial catheter application.

\section{Ultrasound}

The images taken during ultrasonographic examinations are presented in Figure 6A-C. In the ultrasound examinations, the facial vein was identified behind the groove for the facial artery in the submandibular triangle. Pressure application was useful for the segregation of venous structures from arterial structures. When the pressure was applied, the veins collapsed but no significant decrease was observed in the vascular width of the arteries. Identification of the carotid artery, internal jugular vein, common facial vein, and the submandibular vein was feasible by following the facial vein. According to the venous anatomical information obtained from the ultrasonography, no modification was needed for the recommended approach.

\section{DISCUSSION}

In our study, via anatomical dissection, sufficient information about trace and surrounding anatomical structures was obtained about the facial vein, submental vein, and retromandibular vein, which are the suppliers of the common facial vein that drains to the internal jugular vein. Based on the information obtained, we concluded that the catheter application was appropriate via the common facial vein after an incision $2 \mathrm{~cm}$ below the mandibular corpus using two reference points: the groove for the facial artery and the angulus mandibula. According to the results of our study, it would be possible to obtain knowledge about the venous vascular structure with examinations before the application and, accordingly, if needed to modify the incision location for surgical intervention. 

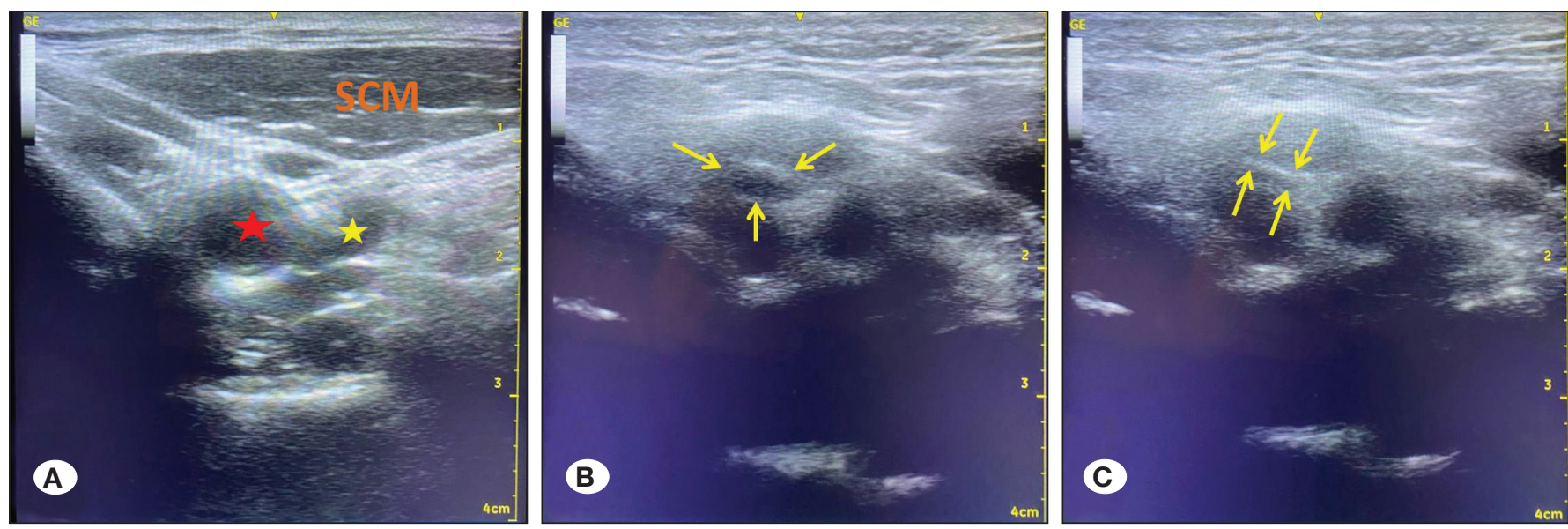

Figure 6: Cervical ultrasonography images for vascular structures. A) In the sternocleidomastoid area, under the sternocleidomastoid muscle (SCM), the carotid artery (red star) and internal jugular vein (yellow star) were observed. B) The common facial vein in the submandibular triangle is indicated by yellow arrows. C) The common facial vein that collapsed after pressure administration with the ultrasound probe is shown by yellow arrows.

VA shunt applications became popular as a result of the developments in shunt materials in the early 1950s $(17,25)$. However, in the following decades, due to the determination of major complications and procedural difficulties, and improvements in the placement of distal catheters in the peritoneal cavity, VA shunt procedures have started to lose their popularity $(2,15)$. Eventually, in the early 80 s VA shunts applications started to become uncommon procedures. In the following decades, neurosurgeons have become less familiar with this procedure and the anatomy of this region. On the other hand, atrial placement of the distal catheter is still a preferable alternative when the peritoneum is not available. In addition to pathological conditions such as peritonitis, malignancy, and malabsorption $(18,20)$, VA shunts are also a treatment alternative in some physiological conditions such as increased intraabdominal pressure due to an enlarged uterus during pregnancy (7). Despite the prevalent impression that VA shunt placement is associated with high complication rates and operative difficulties, some studies have suggested that atrial catheter placements are as reliable as peritoneal catheters $(1,19)$. For these reasons, a study that focused on increasing familiarity with the anatomy and reducing operative difficulties for VA shunt applications such as in our study, would be beneficial.

Accurate placement of the atrial catheter is crucial to prevent severe complications related to VA shunts. Delicate planning before surgery provides increased potential for proper placement of the catheter. The value of using ultrasonographicguided techniques to place an atrial catheter has been demonstrated $(9,20)$. Ultrasound facilitates visualization of the venous vascular structures with distinguishing from arterial vascular structures. Higher calibration and recordable compression with pressure can be observed in veins. In our study, identifying venous structures, such as the internal jugular vein, common facial vein, and facial vein, were achievable via ultrasound. According to these results, no modification was needed in the recommended surgical approach. However, if needed, any requirement for modification of the skin incision could be determined via ultrasonography during surgical planning.

The facial vein, common facial vein, and internal jugular vein are radiologically identifiable vascular structures. MR imaging is a frequently used non-invasive method to analyze the vasculature (12). Evaluating the venous vascular structures is possible with non-contrast MR imaging. Vessel diameter measurements are feasible with MR venography in the cervical region (8). Whether the veins are of suitable width for catheter application can be predicted with venography before the procedure (22). Identifying structures such as the facial vein, common facial vein, and internal jugular vein were possible with the venography performed in our study. No modifications were required in the recommended surgical approach according to the results of the MR venography. If the venous diameter is not considered sufficient for catheter application, the incision can be modified to reach distal venous structures and the incision location can be changed in the horizontal plane according to the location of the venous structures.

Mislocated migration is one of the distal catheter complications of VA shunt procedures. Most migrations occur in the intracardiac area or in the proximal intravascular space (23). However, retrograde (5) or extra-vascular migrations (14) have also been reported. The course of the common facial vein provides an appropriate joining angle with the internal jugular vein for cannulating this vein. This provides reduced risk of retrograde migration during cannulation.

Performing a VA shunt distal catheter application via the facial vein is not appropriate in patients who have had surgery previously in the cervical region (11). In patients with a cervical surgery history, such as tracheostomy, thyroid surgery, and previous VA shunt applications, a more distal venous structure, such as the cephalic vein, can be selected for atrial catheter application (11). In our study, those who had no previous cervical surgery history were included in the cadaver selection. 


\section{CONCLUSION}

In this study, we showed that the common facial vein, draining into the internal jugular vein, is suitable for atrial catheter applications in most VA shunt surgeries. For such applications, a surgical approach via an incision $2 \mathrm{~cm}$ below the mandibular corpus between vertical projections of two reference points: the groove for the facial artery and angulus mandibulae, was recommended. Also, examinations on venous anatomy performed with non-invasive methods, such as MR venography and ultrasound, before the surgical intervention increases the success rate of the procedure.

\section{ACKNOWLEDGMENTS}

We thank to Ms. Rabia Turkkolesi for drawing the illustrative figure (Figure 3) that used in our study.

\section{REFERENCES}

1. Akhtar N, Khan AA, Yousaf M: Experince and outcome of ventricular-atrial shunt: A multi centre study. J Ayub Med Coll Abbottabad 27:817-820, 2015

2. Ames $\mathrm{RH}$ : Ventriculo-peritoneal shunts in the management of hydrocephalus. J Neurosurg 27:525-529, 1967

3. Ben-Ami R, Navon-Venezia S, Schwartz D, Carmeli Y: Infection of a ventriculoatrial shunt with phenotypically variable Staphylococcus epidermidis masquerading as polymicrobial bacteremia due to various coagulase-negative staphylococci and Kocuria varians. J Clin Microbiol 41: 2444-2447, 2003

4. Bergsneider M, Miller C, Vespa PM, Hu X: Surgical management of adult hydrocephalus. Surgery 62:643-660, 2008

5. Cowan MA, Allen MB: Retrograde migration of the venous catheter as a complication of ventriculoatrial shunts in adults, Case report. J Neurosurg 35:348-350, 1971

6. Craven C, Asif H, Farrukh A, Somavilla F, Toma AK, Watkins $\mathrm{L}$ : Case series of ventriculopleural shunts in adults: A singlecenter experience. J Neurosurg 126:2010-2016, 2017

7. Di Muro L, Pallini R, Pietrini D, Colizzi C, Denaro L: Minimally invasive echo-guided placement of the cardiac tube in a ventriculoatrial shunt during pregnancy: Technical note. Neurosurgery 61:E398, 2007

8. Dillman JR, Trout AT, Merrow AC, Moore RA, Rattan MS, Crotty EJ, Fleck RJ, Yoneyama M, Wang H, Tkach JA: Noncontrast three-dimensional gradient recalled echo Dixonbased magnetic resonance angiography/venography in children. Pediatr Radiol 49:407-414, 2019

9. Ellegaard L, Mogensen S, Juhler M: Ultrasound-guided percutaneous placement of ventriculoatrial shunts. Child's Nerv Syst 23:857-862, 2007

10. Emery JL, Hilton HB: Lung and heart complications of the treatment of hydrocephalus by ventriculoauriculostomy. Surgery 50:309-314, 1961
11. Erdogan H, Altun A, Kuruoglu E, Hilmi Kaya A, Dagcinar A: Difficulties of distal catheter insertion of ventriculoatrial shunting in infants and little children. Turk Neurosurg 28:663666, 2018

12. Escott EJ, Branstetter BF: It's not a cervical lymph node, it's a vein: CT and MR imaging findings in the veins of the head and neck. Radiographics 26:1501-1515, 2006

13. Gopal V, Peethambaran A: Rare sequelae following ventriculoatrial shunt: Case report and review of literature. Asian J Neurosurg 11: 173, 2016

14. Hung CC, Chuang HY, Lin HL, Chu YT, Cheng CH: Intramuscular migration of venous catheter as a rare complication of ventriculoatrial shunt: Case report and literature review. J Neurol Surg A Cent Eur Neurosurg 78:412-416, 2017

15. Ignelzi RJ, KirschWM: Follow up analysis of ventriculoperitoneal and ventriculoatrial shunts for hydrocephalus. J Neurosurg 42:679-682, 1975

16. Kahle KT, Kulkarni AV, Limbrick DD, Warf BC: Hydrocephalus in children. Lancet 387:788-799, 2016

17. Konar SK, Maiti TK, Bir SC, Kalakoti P, Nanda A: Robert H. Pudenz (1911-1998) and ventriculoatrial shunt: Historical perspective. World Neurosurg 84:1437-1440, 2015

18. Liu A, Sankey EW, Jusué-Torres I, Patel MA, Elder BD, Goodwin CR, Hoffberger J, Lu J, Rigamonti D: Clinical outcomes after ventriculoatrial shunting for idiopathic normal pressure hydrocephalus. Clin Neurol Neurosurg 143:34-38, 2016

19. McGovern RA, Kelly KM, Chan AK, Morrissey NJ, Mckhann GM: Should ventriculoatrial shunting be the procedure of choice for normal-pressure hydrocephalus? Clinical article. J Neurosurg 120:1458-1464, 2014

20. Metellus P, Hsu W, Kharkar S, Kapoor S, Scott W, Rigamonti $D$ : Accuracy of percutaneous placement of a ventriculoatrial shunt under ultrasonography guidance: A retrospective study at a single institution: Technical note. J Neurosurg 110:867870,2009

21. Nakayama S, Yamashita M, Osaka Y, Isobe T, Izumi H: Right internal jugular vein venography in infants and children. Anesth Analg 93:331-334, 2001

22. Nguyen HS, Turner M, Butty SD, Cohen-Gadol AA: Migration of a distal shunt catheter into the heart and pulmonary artery: Report of a case and review of the literature. Child's Nerv Syst 26:1113-1116, 2010

23. Norin H, Pikwer A, Fellert F, Åkeson J: Internal jugular dimensions and common carotid overlapping assessed in a cross-sectional study by ultrasonography at three neck levels in healthy volunteers. J Vasc Access 18:69-72, 2017

24. Nulsen FE, Spitz EB: Treatment of hydrocephalus by direct shunt from ventricle to jugular vain. Surg Forum 1951:399403, 1951

25. Reddy GK, Bollam P, Caldito G: Long-term outcomes of ventriculoperitoneal shunt surgery in patients with hydrocephalus. World Neurosurg 81:404-410, 2014 